\title{
Time-of-Flight study of molecular beams extracted from the ISOLDE RFQ cooler and buncher
}

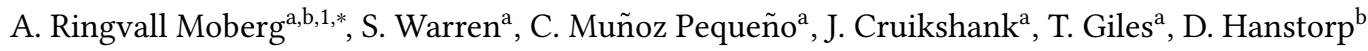 \\ ${ }^{a}$ CERN, 1211 Geneva, Switzerland \\ ${ }^{b}$ Department of Physics, University of Gothenburg, SE-412 96 Gothenburg, Sweden
}

\begin{abstract}
Molecular beams injected into the ISOLDE Radio-Frequency Quadrupole cooler and buncher (RFQcb), ISCOOL, have been studied under varying conditions using a new Time-of-Flight (ToF) detector. When a beam of molecules is injected into the RFQcb and interacts with the buffer gas, collisional dissociation processes may occur. In this study, two different beams of molecules, $\mathrm{CO}^{+}$ and $\mathrm{N}_{2}^{+}$, were separately injected into ISCOOL, and two different buffer gases (pure helium or a 90:10 mixture of helium and neon) were used. The radio-frequency of the RFQcb was varied as the molecules, along with the fragments from the dissociation processes, were extracted from ISCOOL and studied using the new Time-of-Flight detector. The main finding of this work is that the rates for molecular dissociation within ISCOOL were very small for both $\mathrm{CO}^{+}$and $\mathrm{N}_{2}^{+}$, with the largest rates found for $\mathrm{CO}^{+}$.
\end{abstract}

Keywords: ISOLDE, Molecular dissociation, RFQcb, ISCOOL

\section{Introduction}

ISOLDE at CERN is one of the largest ISOL-facilities in the world where radioactive isotopes are produced and studied within many different areas of science [1]. One of the two beamlines at ISOLDE, the High Resolution Separator (HRS), includes a Radio-Frequency Quadrupole cooler and buncher (RFQcb) called ISCOOL [2 3]. The main purpose of a RFQcb is to cool the ions by collisional impact between the ions and a buffer gas. This technique improves the quality of the beam 10 by reducing its transverse emittance before sending it to the experiments [4]. Ions are extracted from ISCOOL either continuously or in a bunched mode. In the latter case, the ions are trapped in the last section of the RFQcb before being extracted. The trapped ions can then be studied using a Time-of-Flight ${ }_{40}$ detector.

In order to extend the number of isotopes and molecules which can be delivered at ISOLDE it is necessary to investigate the processes inside the RFQcb. Molecular ions will be cooled, which is the initial intention of the RFQcb, but they 20 might also dissociate via collisional impact with the buffer gas. The process of molecular dissociation is of great interest in medical applications, for example within CERN-MEDICIS and the MEDICIS-Promed project, where ${ }^{11} \mathrm{C}$ is studied as a possible treatment ion for hadron therapy [5]. In this case, carbon ${ }_{50}$ 25 is extracted from the target material as carbon monoxide [6] since this molecule is more volatile than the atom. The $\mathrm{CO}^{+}$ molecule then needs to be dissociated before it can be used for medical purposes. In other cases, it is a pure ion or molecular

\footnotetext{
${ }^{*}$ Corresponding author

Email address: annie.ringval1 .moberg@cern.ch (A. Ringvall Moberg)
}

beam that is of interest [7]. In such a case, molecular dissociation in the RFQcb may produce contaminating atomic species.

The objective of this study is to investigate whether or not molecular dissociation occurs in the RFQcb. We have investigated this effect in ISCOOL using $\mathrm{CO}^{+}$and $\mathrm{N}_{2}^{+}$ion beams. This is done by varying the radio-frequency and the buffer gas composition inside the RFQcb and observing the mass spectra of the emitted ions.

\section{Experimental set-up and procedure}

The molecular ion beams were produced by injecting neutral molecules in gas form into a FEBIAD ion source [8] where the molecules were ionized and extracted with a potential difference of $40 \mathrm{kV}$. The ion beam was then mass selected and injected into ISCOOL. The RFQcb was filled with ions in $20 \mu \mathrm{s}$ - $5 \mathrm{~ms}$ pulses at a repetition rate of $5 \mathrm{~Hz}$. This was achieved by switching a beam gate placed between the ion source and the RFQcb. Throughout the tests, the cooling time was kept at 5 $\mathrm{ms}$, whereafter the ions were ejected by lowering the potential on the exit electrode of the RFQcb.

Before the molecular ions were injected into ISCOOL they were decelerated in the injection region to an energy of $200 \mathrm{eV}$. ISCOOL was operated in a bunched mode with two different buffer gases. Either pure helium or a mixture of helium and neon (90:10) was used in order to get different center of mass energies in the collisions between the molecules and the atoms in the gas. The collision energy for a head-on impact with $\mathrm{He}$ and $\mathrm{Ne}$ was $25 \mathrm{eV}$ and $83 \mathrm{eV}$ respectively. This is well above the dissociation energies for both $\mathrm{CO}^{+}(8.33 \mathrm{eV})[9]$ and $\mathrm{N}_{2}^{+}(8.712 \mathrm{eV})$ [10]. The pressure inside the RFQcb was first adjusted to give the best transmission for ions in the mass region 
of interest. Thereafter, it was maintained constant throughout

${ }_{60}$ the experiment. The pressure cannot be measured inside the ${ }_{115}$ RFQcb. Instead, a gauge placed just outside the RFQcb showed a relative pressure of $2.5 \cdot 10^{-6}$ mbar.

The radio-frequency field allows ions within a certain mass range to be transmitted through the RFQcb. The radio-

${ }_{65}$ frequency applied to the RFQcb was altered between $680 \mathrm{kHz}$ and $780 \mathrm{kHz}$, in steps of $10 \mathrm{kHz}$, in order to cover the mass region of interest. However, we did not scan the frequency all the way to match mass 28 , i.e. that of $\mathrm{CO}^{+}$and $\mathrm{N}_{2}^{+}$, since the ${ }^{120}$ signal from the primary beam then would completely saturate

70 the detector. Hence, the height of the peak at mass 28 appears much lower than the actual signal.

The detector used during these tests is a new Time-of-Flight detector which is based on secondary electron emission tech- 125 niques and uses a Multi Channel Plate (MCP) detector to create

75 a highly sensitive detector with sub nanosecond timing resolution [11]. It is located in the Central beamline of ISOLDE 10 meters downstream ISCOOL, making it suitable for studies of the time structure and mass spectrum of ion bunches ex-130 tracted from the RFQcb.

\section{3. Results}

The mass range that is transmitted through the RFQcb ${ }^{135}$ is strongly dependent on the applied radio-frequency field. Therefore, an experimental procedure was applied where the ToF spectra for a number of different frequencies were ${ }_{85}$ recorded. However, it is the total mass spectra that is of interest, and we therefore present our results in the form of en- 140 velopes of all the frequencies.

The measured ToF was converted to mass spectra by using the well known peaks of $\mathrm{C}^{+}, \mathrm{O}^{+}$and $\mathrm{CO}^{+}$as calibration

90 lines where the leading edge of the full width at half maximum (FWHM) was used as the reference point.

Fig. 1 shows envelopes of four mass scans when injecting $\mathrm{CO}^{+}$and $\mathrm{N}_{2}^{+}$into ISCOOL. The number of molecules injected into the RFQcb for each radio-frequency scan is shown in the

${ }_{95}$ upper left corner of each graph. It should be noted that the data shown in Fig. 11 1 was recorded using an ion beam two orders 150 of magnitude larger than used in Fig. 1 $\mathrm{b}-\mathrm{d}$. The vertical lines indicates the masses of $\mathrm{C}^{+}, \mathrm{N}^{+}, \mathrm{O}^{+}, \mathrm{H}_{2} \mathrm{O}^{+}, \mathrm{Ne}^{+}$and $\mathrm{CO}^{+} / \mathrm{N}_{2}^{+}$. The real number of ions corresponding to atomic mass $28\left(\mathrm{CO}^{+}\right.$ 100 and $\mathrm{N}_{2}^{+}$) is, as described above, much higher than shown in the graphs.

The main result of the experiment is that the rate of molecular dissociation in the RFQcb is very small. The primary beam of $\mathrm{CO}^{+}$or $\mathrm{N}_{2}^{+}$completely dominates the extracted ion beam.

105 This peak is so large so we were not able to measure it using the particle detector.

The second largest peaks appear at mass 18, which corresponds to the mass of water. This peak is present in all of the recorded spectra.

Finally, we observe small peaks that correspond to carbon, nitrogen and oxygen. These could, as will be further discussed ${ }_{165}$ in the next section, originate from molecular dissociation inside the RFQcb. Between $4 \cdot 10^{-6}$ and $5 \cdot 10^{-5}$ of the $\mathrm{CO}^{+}$ions in a bunch injected into ISCOOL were detected as $\mathrm{C}^{+}$fragments on the ToF detector.

\section{Discussion}

The main finding of our experiment is that only minute fractions of $\mathrm{CO}^{+}$and $\mathrm{N}_{2}^{+}$are dissociated in the RFQcb. At first, this might seem somewhat surprising since the energy in the collision between the molecular ion and the buffer gas is much higher than the dissociation energy of the molecular ion. However, the dissociation will mainly occur by a collision where an electronic excitation brings the molecular ion to an antibonding state in which it dissociates. For example, Fig. 4 of Ref. [12], shows that excitation from the ground state to the first anti-bonding state in $\mathrm{CO}^{+}$requires an energy almost twice its dissociation energy. The same can be seen for $\mathrm{N}_{2}^{+}$in Fig. 1 of Ref. [13]. Further, the full center of mass collision energy will only be obtained in head-on collisions, which only occur for a small fraction of the collisions. Instead, the molecular ions will, as they enter the RFQcb, undergo multiple collisions where they gradually lose their energy such that they eventually cannot be dissociated. Hence, molecular dissociation will only occur at the entrance of the RFQcb. It should then be pointed out that only dissociations at the entrance of the cooler will be detected since molecules dissociated inside the cooler would require one specific radio-frequency to guide the molecular ions into the cooler and a different radio-frequency to bring the lighter fragments out.

In the $\mathrm{CO}^{+}$measurements (Fig. $1 \mathrm{a}$ and $1 \mathrm{p}$ ) we do observe a small peak of carbon, whereas there is no peak present at the atomic mass 16, which would correspond to atomic oxygen. This is in agreement with the previous findings of Friedländer [14] and Garcia [12] who observed that dissociation resulting in $\mathrm{C}^{+}+\mathrm{O}$ dominates over $\mathrm{C}+\mathrm{O}^{+}$. This is also consistent with the larger ionization energy of oxygen $(13.6 \mathrm{eV})$ vs. carbon $(11.3 \mathrm{eV})$. We do also observe a small nitrogen peak which most likely is originating from $\mathrm{N}_{2}$ contamination in the cooler. Finally, we observe a peak at mass 18 . This is expected to be $\mathrm{H}_{2} \mathrm{O}^{+}$as it is a common vacuum contaminant, however the width of the peak is larger than expected which may be due to space-charge related effects or the presence of an additional mass. One possible candidate for this could be $\mathrm{Ne}^{+}$.

In Fig. 1 and $1 \mathrm{~d}$, which are the spectra related to the measurements of $\mathrm{N}_{2}^{+}$molecules, we observe that the atomic mass 12 has been severely diminished, as expected. When comparing the results of $\mathrm{CO}^{+}$and $\mathrm{N}_{2}^{+}$we observe larger dissociation rates of $\mathrm{CO}^{+}$even though the dissociation energies for the two molecules are relatively similar. The difference in dissociation rates may be due to the difference in the potential energy curves for the two molecules. It might, for instance be possible that $\mathrm{CO}^{+}$molecule is excited into a bound state which couples adiabatically to another state which, in turn, dissociates.

There are no obvious differences observed in the data recorded using the two different buffer gases (Figs. 17 vs. 1b and Figs. 11c vs. 1 d). This is most likely an effect of the relatively low concentration of neon in the gas mixture. 

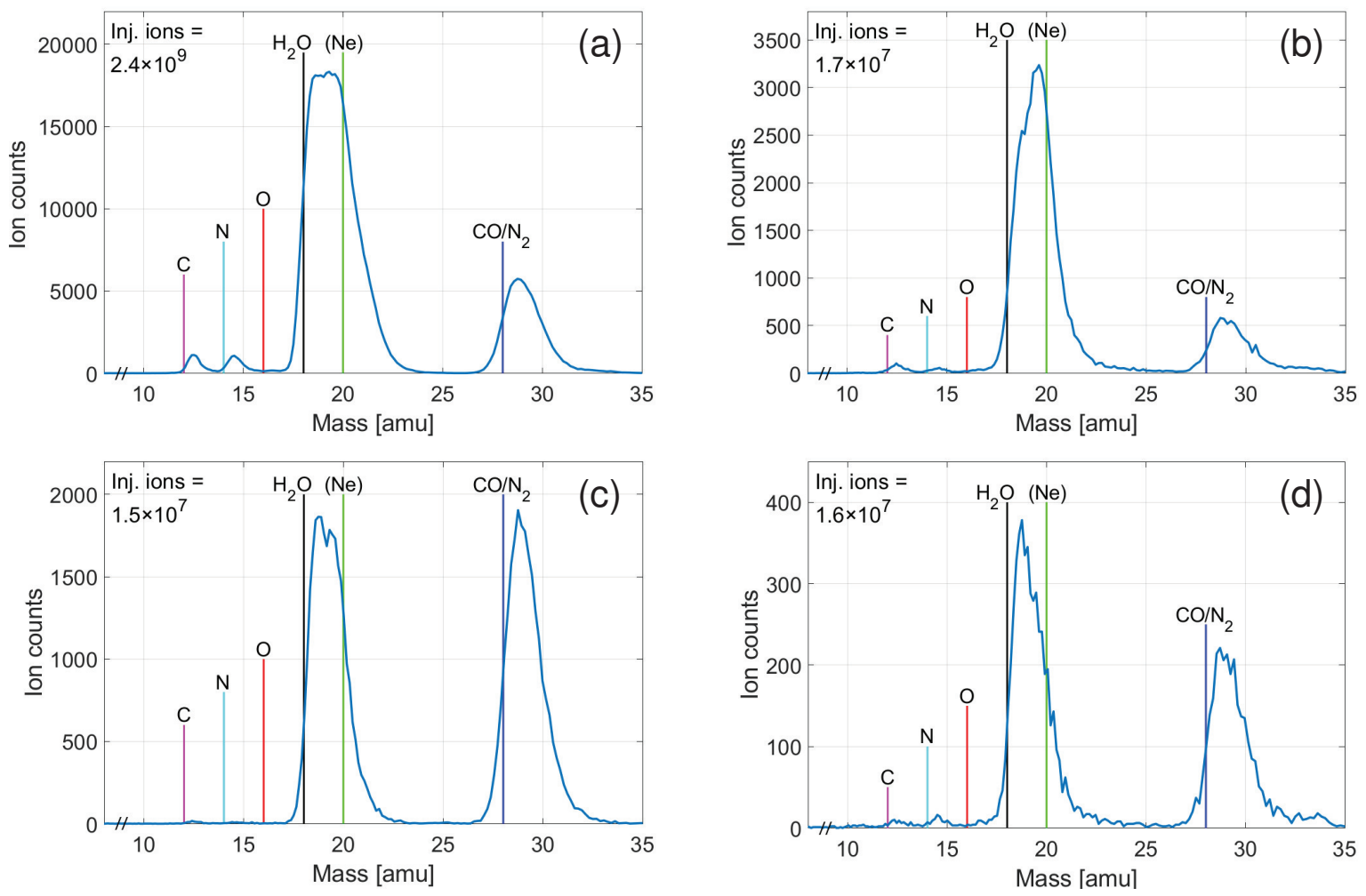

Figure 1: Graphs showing the envelopes of four mass scans when injecting molecular beams of (a) $\mathrm{CO}^{+}$into $\mathrm{He}$, (b) $\mathrm{CO}^{+}$into $\mathrm{He}$ :Ne buffer gas, (c) $\mathrm{N}_{2}^{+}$into He and (d) $\mathrm{N}_{2}^{+}$into He:Ne buffer gas. The vertical lines indicate to the masses of $\mathrm{C}^{+}, \mathrm{N}^{+}, \mathrm{O}^{+}, \mathrm{H}_{2} \mathrm{O}^{+}$, $\mathrm{Ne}^{+}$and $\mathrm{CO}^{+} / \mathrm{N}_{2}^{+}$with respect to the leading edge of the envelopes. The number of ions injected for each radio-frequency scan is noted in the upper left corner of the graphs.

The result presented in this work shows that a RFQcb is not a very efficient way to produce $\mathrm{C}^{+}$from $\mathrm{CO}^{+}$since only $4 \cdot 10^{-6}$ to $5 \cdot 10^{-5}$ of the molecular ions injected into ISCOOL dissociate. Instead, the work shows that it might be possible to use a RFQcb to cool and bunch molecular ion beams with only very ${ }^{195}$ small losses. One application may be within the MEDICISPromed project where possible schemes of injecting radioactive ion beams into a synchrotron-based medical accelerator have been investigated [7].

\section{Conclusion}

The Time-of-Flight spectra of cooled molecular ion beams extracted from ISCOOL have been investigated. It is possible to observe molecular dissociation within ISCOOL when containing either pure helium buffer gas or a gas mixture of helium and neon (90:10). Molecular dissociation within ISCOOL was observed for both $\mathrm{CO}^{+}$and $\mathrm{N}_{2}^{+}$, with the largest rates found for $\mathrm{CO}^{+}$. However, the fraction of molecular ions that dissociate inside the RFQcb is orders of magnitude smaller than the fraction that remain in the molecular form. Hence, this work indicates that molecular dissociation in a RFQcb is not a very efficient way of producing beams containing only atomic ions. On the contrary, the results indicate that the RFQcb can be

\section{Acknowledgement}

We would like to acknowledge the ISOLDE technical team for their contributions as well as Alessandro Masi for providing the data acquisition system.

This research project has been supported by a Marie Skłodowska-Curie innovative training network fellowship of the European commission's horizon 2020 program under contract number 642889 MEDICIS-PROMED and by the Swedish

\section{References}

[1] R. Catherall et al., J. Phys. G: Nucl. Part. Phys. 44 (9) (2017) 094002.

[2] C. Babcock et al., Nucl. Instrum. Meth. Phys. Res. B 317 (2013) 484-487.

[3] A. Jokinen et al., Nucl. Instrum. Meth. Phys. Res. B 204 (2003) 86-89.

[4] A. Kellerbauer et al., Nucl. Instrum. Meth. Phys. Res. A 469 (2001) 276285

[5] R. S. Augusto et al., Nucl. Instrum. Meth. Phys. Res. B 376 (2016) 374-378.

[6] S. Stegemann et al., To be submitted to Nucl. Instrum. Meth. Phys. Res. B (current volume) .

[7] J. Pitters et al., CERN Internal Note, CERN-ACC-NOTE-2018-0078.

[8] R. Kirchner et al., Nucl. Instrum. Methods 139 (1976) 291-296.

[9] R. R. Reddy et al., J. Astrophys. Astr. 11 (1) (1990) 67-72.

10] K. P. Huber et al., Van Nostrand Reinhold Company, New York, 1979.

[11] S. Warren et al., To be published.

[12] G. García et al., Int. J. Mass Spectroscopy 261 (1) (2007) 53 - 56.

[13] D. C. Cartwright et al., J. Phys. B: Atom. Molec. Phys 8 (6) (1975) 100-103.

[14] E. Friedländer et al., Z. Physik 76 (1932) 60-69. 